Check for updates

Cite this: RSC Adv., 2017, 7, 31133

Received 23rd April 2017

Accepted 11th June 2017

DOI: $10.1039 / c 7 r a 04549 b$

rsc.li/rsc-advances

\section{A multifunctional nanoplatform based on mesoporous silica nanoparticles for imaging- guided chemo/photodynamic synergetic therapy $\uparrow$}

\begin{abstract}
Yadan Liu, (D) ab Xiaolin Liu, ${ }^{\mathrm{a}}$ Yue Xiao, ${ }^{\mathrm{ab}}$ Fangman $\mathrm{Chen}^{\mathrm{ab}}$ and Fangnan Xiao (D) *a
Multifunctional nanoplatforms based on mesoporous silica nanoparticles (MSNs) have recently shown great promise in drug delivery and therapy. Herein, a multifunctional nanoplatform based on MSNs is fabricated by a modified micro-emulsion method for drug delivery and imaging-guided chemo/photodynamic synergistic therapy. Carbon dots (C-dots) and a photosensitizer, rose bengal (RB), are embedded in the core/shell structured MSNs to form MSNaC-dots/RB nanoparticles, in which C-dots can serve as a fluorescence probe to achieve cell fluorescence imaging and RB can generate singlet oxygen to perform photodynamic therapy. MSNaC-dots/RB nanoparticles can efficiently prevent the selfaggregation-induced quenching of photosensitizer molecules, which facilitates a large production of cytotoxic singlet oxygen and thus an enhanced PDT efficacy. Furthermore, a remarkable chemo/ photodynamic synergistic anti-tumor effect was achieved with MSNaC-dots/RB nanoparticles loaded with doxorubicin (DOX) under green light irradiation. In addition, a significant bacterial inhibitory effect has been achieved by the antimicrobial assay via loading ampicillin in the MSNs@C-dots/RB nanoparticles, thereby demonstrating the versatility of this multifunctional nanoplatform. The results revealed that this MSN-based chemo/photodynamic synergistic nanoplatform has great promise in imaging assisted cancer therapy and bacteria inhibition.
\end{abstract}

\section{Introduction}

With the development of biomedicine, the establishment and application of multifunctional therapy nanoplatforms has become a new trend for cancer therapy., ${ }^{1,2}$ Nanoplatforms integrated with diagnostic and therapeutic functions (e.g., multimodal imaging, targeted delivery, synergistic therapy) have been widely researched..$^{3,4}$ For example, Liu et al. synthesized a multifunctional polymeric nano-micelle system, containing the photosensitizer chlorin e6 (Ce6), $\mathrm{Gd}^{3+}$ and the near-infrared (NIR) dye, IR825. The use of these micelles as a contrast agent achieved triple modal imaging of tumors in vivo. The combination of photothermal and photodynamic therapies has remarkably brought about both in vitro and in vivo synergistic anti-tumor effects. ${ }^{5}$ In recent years, various materials, such as metals, silica, graphene, polymers, metal organic frameworks (MOF) and clay, have been used for constructing multifunctional drug systems. ${ }^{6-13}$ Among the current nanoplatforms, the

${ }^{a}$ State Key Laboratory of Structural Chemistry, Fujian Institute of Research on the Structure of Matter, Chinese Academy of Sciences, Fuzhou, Fujian 350002, China. E-mail: fnxiao@firsm.ac.cn

${ }^{b}$ College of Life Sciences, Fujian Agriculture and Forestry University, Fuzhou, Fujian 350002, China

$\dagger$ Electronic supplementary information (ESI) available. See DOI: $10.1039 / \mathrm{c} 7 \mathrm{ra} 04549 \mathrm{~b}$ mesoporous silica nanoparticles (MSNs) have been developed as a promising candidate for multifunctional cancer therapy owing to their high surface area, large pore volume with tunable pore sizes, facile surface modification, high light transparency and good biocompatibility. ${ }^{714-17}$ For example, MSN@ $\mathrm{Fe}_{3} \mathrm{O}_{4}-$ FITC nanoparticles have been developed for MR and luminescence imaging of NIH 3 T3 fibroblast cells. ${ }^{18}$ PEG-silica nanorattles have been designed as nanocarriers of doxorubicin (DOX) for cancer therapy and show significantly increased liver tumor inhibition rate and decreased toxicity. ${ }^{19}$ Folate-MSNs exhibit escaping endosomal entrapment and enhance the HeLa cellular uptake of these materials. ${ }^{20}$

Rose bengal (RB) is a well-known green light-activated photosensitizer that shows absorption bands in the visible range (480-550 nm) and high singlet oxygen quantum yield $\left(\Phi\left({ }^{1} \mathrm{O}_{2}\right)=0.75\right){ }^{21-23} \mathrm{RB}-\mathrm{MSN}$ nanoplatforms have been reported for photodynamic therapy. ${ }^{24-26}$ For example, RB can be covalently bound to MSN by thiolene click chemistry method and showed highly effective antibacterial activity. ${ }^{23}$ RB-modified MSN displayed high photostability and efficiency in the photoproduction of singlet oxygen. ${ }^{21,22}$ PEG-modified RB-loaded MSN exhibits about 10 times apoptosis efficiency than that of free $\mathrm{RB} .{ }^{26}$ Although great progress has been achieved in the development of MSN-based nanoplatforms, several challenges still exist in multifunctional MSNs as drug systems for clinic application. First, the complexity of components in a single 
nanoparticle will bring a mutual interference between dyes and/ or nanoparticles, which reduces diagnostic and therapeutic efficacy. Therefore, many studies are undergoing to design various structures such as hollow, core/shell to control these side-effects..$^{14,17,19}$ Second, the incorporation of multiple types of drugs into one nanoparticle makes it difficult to obtain high encapsulation efficiency for each drug. It could also influence the drug release kinetic and release mode. Hence, various methods have been developed including the doping of silica with drugs during preparation and co-condensation of silica source (e.g., organosilane) ${ }^{27-29}$ For example, the photosensitizer porphyrin derivatives can be covalently encapsulated inside MSN for photodynamic therapy. ${ }^{30}$ The porphyrin-MSN can also load DOX in the pore of MSN for chemo/photodynamic therapy. ${ }^{31}$ Third, many methods involving multiple steps are costly. ${ }^{7,19,20,32}$ Therefore, MSN-based nanoplatforms are still in the early stage for applications.

As the emerging fluorescence nanomaterial, carbon dot (Cdot) has unique properties, such as the tunable fluorescence emission, chemical inertness and excellent biocompatibility. ${ }^{33-38}$ They are becoming widely used to facilitate the construction of multifunctional medical nanomaterials. . $^{34,39-41}$ However, due to their aggregation-induced luminescence quenching property, the photoluminescence effect of C-dots was seriously destroyed at the high concentrations of the Cdots, leading to serious hindrance of their application in bioimaging. ${ }^{42}$ Hence, many materials have been used for dispersion and encapsulation of C-dots to avoid the undesirable effects. ${ }^{43-47}$ For example, the fluorescent of carbon dot-MSNs can be enhanced by $\mathrm{Ag}$ based on the metal-enhanced fluorescence effect. ${ }^{48}$ In addition, highly luminescent organosilanefunctionalized C-dots were synthesized, ${ }^{46}$ which were demonstrated to be used for $\mathrm{Cu}^{2+}$ detection. ${ }^{49}$ Because the stability and biocompatibility were greatly improved by silica-coating. ${ }^{44,45,50-52}$ C-dots@MSN nanoparticles have been developed for molecular delivery, where C-dots on the surface of MSNs were used as cell imaging agent, and the large pore volume of MSNs can load the chemotherapeutic drugs for cancer therapy. ${ }^{53}$

In this study, a multifunctional nanoplatform based on MSNs carrying C-dots and a photosensitiser rose bengal (RB) was designed for drug delivery, synergistic chemo/ photodynamic cancer therapy and inhibition of bacteria. This nanoplatform consisted of two mesoporous layers with C-dots and RB embedded in different layers, respectively. C-dots were used for cell fluorescence imaging. RB was introduced to achieve the effective photodynamic therapy and fluorescence imaging. The MSNs embedding C-dots systems maintained the excellent fluorescence properties of C-dots, and benefited further application of bioimaging and therapy. Meanwhile, the epitaxial growth characteristic of the mesoporous silica shell on MSNs avoided the mutual interference between C-dots and RB to realize photodynamic therapy (PDT). Furthermore, DOX loaded in the pore of MSNs for chemotherapy. The results exhibited significantly increasing cancer therapy in human lung cancer cell line NCI-H1299 (H1299) due to the synergistic chemo/photodynamic effect. And this MSN@C-dots/RB multifunction nanoplatform also showed good bacteria (E. coli) inhibition and thereby could achieve more effective therapeutic effects in cancer therapy, since frequently the bacterial infection contamination accompanying cancer progression can injure the immune system and deteriorate cancers..$^{54}$ Compared to other nanoplatforms, our design had the advantages to avoid the mutual interference between C-dots and RB, and guarantee an economic strategy for synergistic effects on cancer therapy and bacteria inhibition.

\section{Experimental}

\section{Chemicals and materials}

Sodium hydroxide ( $\mathrm{NaOH})$, citric acid, urea, rose bengal (RB), tetraethylorthosilicate (TEOS), cetyltrimethylammonium chloride (CTAC), 1,3-diphenylisobenzofuran (DPBF), triethanolamine (TEA), dimethylformamide (DMF), and ethanol were purchased from Sinopharm Chemical Reagent Co., China. Hoechst 33342 and 1-octadecene were purchased from SigmaAldrich (China). All the chemical reagents were of analytical grade and used as received without further purification.

\section{Synthesis of carbon dots}

The C-dots were prepared by previous methods. ${ }^{35}$ In detail, citric acid $(1 \mathrm{~g})$, urea $(2 \mathrm{~g})$ and DMF $(10 \mathrm{~mL})$ were added into a $20 \mathrm{~mL}$ Teflon stainless steel autoclave and reacted at $160{ }^{\circ} \mathrm{C}$ for $6 \mathrm{~h}$. The obtained solution was mixed with $\mathrm{NaOH}(20 \mathrm{~mL}, 50 \mathrm{mg}$ $\mathrm{mL}^{-1}$ ), and centrifuged at $16000 \mathrm{rpm} \mathrm{min}{ }^{-1}$ for $10 \mathrm{~min}$. The precipitate was redispersed in water, centrifuged. The freezedried product C-dots were got.

\section{Preparation of carbon dots embedded mesoporous silica nanoparticles (MSN@C-dots)}

MSN@C-dots nanoparticles were achieved by a modified microemulsion method. C-dots were doped in the process of MSN@Cdots nanoparticles preparation. The MSNs were prepared via an oil-water biphase stratification approach by using cationic surfactant CTAC as a template, TEOS as a silica source, TEA as a catalyst and organic solvent 1-octadecene or cyclohexane as an emulsion agent. ${ }^{32} \mathrm{~A}$ typical synthesis of the carbon dots doped mesoporous silica nanospheres (MSN@C-dots) was performed as following. Firstly, CTAC solution $(12 \mathrm{~mL}, 25 \mathrm{wt} \%)$, carbon dots solution $\left(2.5 \mathrm{~mL}, 1 \mathrm{mg} \mathrm{mL}{ }^{-1}\right)$ and TEA $(0.09 \mathrm{~g})$ were sequentially added to water $(18 \mathrm{~mL})$ and stirred mildly at $60^{\circ} \mathrm{C}$ for $1 \mathrm{~h}$ in a $50 \mathrm{~mL}$ round bottom flask, then TEOS solution in 1octadecene $(10 \mathrm{~mL}, 20 \mathrm{v} / \mathrm{v} \%)$ was slowly added to the waterCTAC-TEA solution and kept at $60{ }^{\circ} \mathrm{C}$ in an oil bath under a gentle magnetic stirring. Then the reaction was kept at a constant temperature with continuous stirring for $12 \mathrm{~h}$ to obtain the products. After that, the upper phase, 1-octadecene solution was completely removed and the products were collected by centrifugation and washed with ethanol for 4 times to remove the residual reactants. Then, the collected products were extracted with ammonium nitrate $\left(\mathrm{NH}_{4} \mathrm{NO}_{3}\right)$ ethanol solution $(50 \mathrm{~mL}, 0.6 \mathrm{wt} \%)$ at $60{ }^{\circ} \mathrm{C}$ for $12 \mathrm{~h}$ to remove the template, and centrifuged at $16000 \mathrm{rpm} \mathrm{min}{ }^{-1}$ for $10 \mathrm{~min}$. The precipitate was collected, dissolved in ethanol and centrifuged 
twice, and then freeze-dried to get the brown products of MSN@C-dots nanoparticles.

\section{Preparation of MSN@C-dots/RB nanoparticles}

The synthesis of the MSN@C-dots/RB was following with the preparation of MSN@C-dots. At first, CTAC solution (12 mL, 25 wt $\%)$, carbon dots solution $\left(2.5 \mathrm{~mL}, 1 \mathrm{mg} \mathrm{mL}^{-1}\right)$ and TEA $(0.09$ g) were sequentially added to water $(18 \mathrm{~mL})$ and stirred mildly at $60{ }^{\circ} \mathrm{C}$ for $1 \mathrm{~h}$ in a $50 \mathrm{~mL}$ round bottom flask, then TEOS solution in 1-octadecene $(10 \mathrm{~mL}, 20 \mathrm{v} / \mathrm{v} \%)$ was slowly added to the waterCTAC-TEA solution and kept at $60{ }^{\circ} \mathrm{C}$ in an oil bath under a gentle magnetic stirring. Then the reaction was kept at a constant temperature with continuous stirring for $12 \mathrm{~h}$ to obtain the products. Then, the upper phase, 1-octadecene solution was completely removed and the products were collected by centrifugation and washed for several times with ethanol to remove the residual reactants. Then, the collected products, rose bengal solution $\left(1 \mathrm{~mL}, 2 \mathrm{mM} \mathrm{mL}^{-1}\right)$, CTAC solution $(12 \mathrm{~mL}, 25 \mathrm{wt} \%)$ and TEA $(0.09 \mathrm{~g})$ were added to water $(18 \mathrm{~mL})$ and stirred gently at $60{ }^{\circ} \mathrm{C}$ for $1 \mathrm{~h}$ in a $50 \mathrm{~mL}$ round bottom flask. Then TEOS solution in cyclohexane $(10 \mathrm{~mL}, 20 \mathrm{v} /$ $\mathrm{v} \%)$ was slowly added and kept at $60^{\circ} \mathrm{C}$ for another $12 \mathrm{~h}$ for the growth of the RB embedded MSNs shell. The products were collected by centrifugation and washed for several times with ethanol to the residual reactants. Then, the collected products were extracted with a $0.6 \mathrm{wt} \%$ ammonium nitrate $\left(\mathrm{NH}_{4} \mathrm{NO}_{3}\right)$ ethanol solution at $60{ }^{\circ} \mathrm{C}$ for $24 \mathrm{~h}$ to remove the template and centrifuged at $16000 \mathrm{rpm} \mathrm{min}{ }^{-1}$ for $10 \mathrm{~min}$. The precipitate was collected, dissolved in ethanol and centrifuged twice, and then freeze-dried to get the pink products of MSN@C-dots/RB nanoparticles.

\section{Measurement of the loading capacity of photosensitizer in MSN@C-dots/RB nanoparticles}

The content of RB doped in MSN@C-dots/RB nanoparticles was determined by measuring the characteristic emission peak of $\mathrm{RB}$ at $540 \mathrm{~nm}$ for the solution of MSN@C-dots/RB nanoparticles $\left(100 \mu \mathrm{g} \mathrm{mL}{ }^{-1}\right)$. The weight amount of RB embedded in MSN@Cdots/RB nanoparticles could be calculated based on the standard curve derived from the emission spectra of the different concentrations of pure RB solution. The loading capacity of RB was calculated as follows: loading capacity $(\%)=$ (weight amount of RB in MSN@C-dots/RB nanoparticles)/(weight amount of MSN@C-dots/RB nanoparticles) $\times 100 \%$.

\section{Measurement of the release of photosensitizers from MSN@C-dots/RB nanoparticles}

Firstly, MSN@C-dots/RB nanoparticles (4.0 mg) were soaked in PBS buffer $(1 \mathrm{~mL})$ with different $\mathrm{pH}(\mathrm{pH}=5.0,6.0,6.5$, and 7.0) under continuous stirring at $37^{\circ} \mathrm{C}$ in darkness. After soaking for $72 \mathrm{~h}$, the solution of MSN@C-dots/RB nanoparticles was centrifuged at $16000 \mathrm{rpm}$ for $5 \mathrm{~min}$. The corresponding supernatant was then assessed to determine whether the photosensitizers were released into solution by measuring their emission spectra.

\section{Singlet oxygen detection}

A chemical probe 1,3-diphenylisobenzofuran (DPBF) was used to confirm the ${ }^{1} \mathrm{O}_{2}$ generation by detecting its absorption intensity at $415 \mathrm{~nm}$ via UV-Vis spectroscopy. In a typical experiment, DPBF in ethanol $(10 \mu \mathrm{L}, 5 \mathrm{mM})$ was added to ethanol $/ \mathrm{H}_{2} \mathrm{O}$ solution $(50 \mathrm{v} / \mathrm{v} \%)$ containing MSN@C-dots/RB nanoparticles $\left(3 \mathrm{~mL}, 0.1 \mathrm{mg} \mathrm{mL}^{-1}\right)$. The solution was kept in the dark and irradiated with a $540 \mathrm{~nm}$ lamp $\left(300 \mathrm{~mW} \mathrm{~cm}{ }^{-2}\right)$ for $10 \mathrm{~min}$, and the absorption intensity of DPBF at $415 \mathrm{~nm}$ was recorded every $1 \mathrm{~min}$. In the control experiments, DPBF absorption was recorded in MSN@C-dots nanoparticles counterparts or in the absence of $540 \mathrm{~nm}$ irradiation under otherwise identical conditions.

\section{DOX loading and releasing}

MSN@C-dots/RB nanoparticles (12 mg) was mixed with PBS (10 $\mathrm{mL}, \mathrm{pH}$ 7.4) containing $5 \mathrm{mg}$ of DOX. After stirring for $12 \mathrm{~h}$ in the dark, the DOX loaded MSN@C-dots/RB nanoparticles were collected by centrifugation. The samples were washed with PBS for several times. The content of DOX was determined by detecting the absorption spectra of supernatant and original DOX solution at $482 \mathrm{~nm}$. The DOX loading capacity and loading efficiency were calculated as follows: DOX loading capacity $(\mathrm{wt} \%)=($ original DOX - DOX in supernatant $) /($ MSN@C-dots/ $\mathrm{RB}$ nanoparticles) $\times 100 \%$ and DOX loading efficiency (wt $\%)$ $=($ original DOX $-\mathrm{DOX}$ in supernatant $) /($ original DOX $) \times 100 \%$. The DOX releasing experiments were carried out in PBS buffer at different $\mathrm{pH}$ (pH 5.0, 6.0, and 7.0). MSN@C-dots/RB/DOX nanoparticles $(2.0 \mathrm{mg})$ were dispersed in PBS buffer $(2 \mathrm{~mL})$ and then incubated at $37{ }^{\circ} \mathrm{C}$ with gentle shaking in darkness. $100 \mu \mathrm{L}$ of supernatant was taken out after centrifugation at specified time points to detect the absorption spectra at $482 \mathrm{~nm}$. The equal volume of fresh PBS buffer with different $\mathrm{pH}$ was added to keep the total volume constant.

\section{Cell culture and confocal laser scanning microscopy imaging}

Human lung cancer cells (H1299) and human embryo lung fibroblasts cells (HELF) cell lines were purchased from Shanghai Institute of Cell Biology, Chinese Academy of Sciences, and were routinely maintained in RPMI-1640, supplemented with $10 \%(\mathrm{v} / \mathrm{v})$ heat-inactivated fatal calf serum (FCS), penicillin $\left(100 \mathrm{U} \mathrm{mL}^{-1}\right)$, and streptomycin $\left(100 \mathrm{mg} \mathrm{mL}^{-1}\right)$ at $37{ }^{\circ} \mathrm{C}$ under humidified air containing $5 \% \mathrm{CO}_{2}$. $\mathrm{H} 1299$ cells were seeded into culture plates and allowed to adhere for $24 \mathrm{~h}$. After washing with PBS, the cells were incubated in culture medium containing MSN@C-dots/RB nanoparticles at $37^{\circ} \mathrm{C}$ for $2 \mathrm{~h}$ under $5 \% \mathrm{CO}_{2}$ and then washed with PBS sufficiently to remove excess nanoparticles. The cells were subsequently incubated with Hoechst 33342 at RT for $10 \mathrm{~min}$ and washed with PBS. The cell imaging was performed with a laser scanning confocal fluorescence microscope (Leica SP5). The luminescence signals were detected in the green channel (565-590 nm), red channel (590-680 $\mathrm{nm}$ ) and blue channel (450-490 nm), respectively. 


\section{Cell viability assay}

H1299 cells at a density of $5 \times 10^{4}$ cells per milliliter were placed in 96-well plates with a volume of $200 \mu \mathrm{L}$ per well and incubated overnight at $37{ }^{\circ} \mathrm{C}$ under $5 \% \quad \mathrm{CO}_{2}$. The culture medium was carefully removed and the cells were incubated with different concentrations of MSN@C-dots/RB nanoparticles or MSN@C-dots/RB/DOX nanoparticles (0, 10, 30, 50, 80 and $100 \mu \mathrm{g} \mathrm{mL}^{-1}$ ) diluted with fresh culture medium for $4 \mathrm{~h}$. The cells were incubated with fresh culture medium as the control. The cells were then washed twice with sterile PBS before fresh culture medium was added. For PDT experiment, the cells were exposed to $540 \mathrm{~nm}$ irradiation with a power density of $300 \mathrm{~mW}$ $\mathrm{cm}^{-2}$ for $5 \mathrm{~min}$. Then the cells were cultured in $5 \% \mathrm{CO}_{2}$ at $37{ }^{\circ} \mathrm{C}$ for additional $24 \mathrm{~h}$. MTT was subsequently added to the cells followed by incubation at $37^{\circ} \mathrm{C}$ under $5 \% \mathrm{CO}_{2}$ for $4 \mathrm{~h}$, and then the culture medium was carefully discarded and replaced with DMSO. The $\mathrm{OD}_{490}$ value of each well was measured on a multimodal microplate reader (Synergy 4, BioTek). The following formula was applied to calculate the percent inhibition rate of cell growth: cell viability $(\%)=$ (mean of absorbance value of treatment group/mean of absorbance value of control) $\times 100 \%$. Four replicates were run per nanoparticles dose in each cell lines. The same protocol was utilized to determine the cytotoxicity of MSN@C-dots/RB nanoparticles to HELF cells, except that the HELF cells were incubated with nanoparticles for $24 \mathrm{~h}$ without PDT treatment.

\section{Antimicrobial activity measurement of the MSN@C-dots/RB nanoparticles}

For ampicillin storage, the as-obtained MSN@C-dots/RB nanoparticles $(20 \mathrm{mg})$ was mixed with $500 \mu \mathrm{L}$ of ampicillin aqueous solution $\left(100 \mathrm{mg} \mathrm{mL}^{-1}\right)$ under a magnetic stirring at $37{ }^{\circ} \mathrm{C}$ for $12 \mathrm{~h}$ in dark. The excess amount of ampicillin was removed by centrifugation and washing with deionized water for several times. The content of ampicillin was determined by detecting the UV absorption spectra of supernatant and original ampicillin solution at $263 \mathrm{~nm}$. The Amp loading capacity and loading efficiency were calculated as follows: Amp loading capacity $(\mathrm{wt} \%)=($ original Amp - Amp in supernatant)/(MSN@C-dots/ $\mathrm{RB}$ nanoparticles) $\times 100 \%$ and Amp loading efficiency (wt $\%$ ) $=($ original Amp - Amp in supernatant $) /($ original Amp $) \times 100 \%$. Escherichia coli ( $E$. coli, a kind of Gram-negative bacteria) was used as model in our experiment. The $E$. coli was grown in LB medium on a shaking incubator $(170 \mathrm{rpm})$ at $37^{\circ} \mathrm{C}$ overnight for bacterial growth into exponential phase. Bacterial suspensions diluted to $1 \times 10^{4}$ times was treated with different concentrations of MSN@C-dots/RB or MSN@C-dots/RB/Amp with three parallel samples for each group and exposed to $540 \mathrm{~nm}$ irradiation with a power density of $300 \mathrm{~mW} \mathrm{~cm}^{-2}$ for $10 \mathrm{~min}$. Then $E$. coli was grown in LB medium on a shaking incubator (230 rpm) at $37^{\circ} \mathrm{C}$ for $20 \mathrm{~h} .100 \mu \mathrm{L}$ liquids was taken out from the flask at predetermined time points and the values of the optical density (OD) at $600 \mathrm{~nm}$ were record. For agar plate experiments, the bacterial suspensions was diluted $10^{4}$ times and incubated with $100 \mu \mathrm{g} \mathrm{mL}^{-1}$ of MSN@C-dots/RB or MSN@C-dots/RB-Amp on a shaking incubator $(230 \mathrm{rpm})$ at $37^{\circ} \mathrm{C}$ for $1 \mathrm{~h}$. Then $E$. coli was exposed to $540 \mathrm{~nm}$ irradiation with a power density of $300 \mathrm{~mW}$ $\mathrm{cm}^{-2}$ for $10 \mathrm{~min} .100 \mu \mathrm{L}$ of the bacterial suspensions was spread onto the agar plates and the agar plates were then inverted and incubated at $37{ }^{\circ} \mathrm{C}$ for $12 \mathrm{~h}$. Photographs of $E$. coli colonies grown on the agar plates were obtained.

\section{Structural and optical characterization}

Transmission electron microscopy (TEM) measurements were performed on a Tecnai F20 TEM equipped with the energydispersive X-ray spectrum. The hydrodynamic diameter distribution of MSN@C-dots nanoparticles and MSN@C-dots/RB nanoparticles were determined by means of dynamic light scattering (DLS) measurement (Nano ZS ZEN3600, Malvern). $\mathrm{N}_{2}$ sorption/desorption measurements were performed on a Micromeritics ASAP2020M instrument at $77 \mathrm{~K}$. UV-Vis absorption spectra were measured with a Perkin-Elmer Lambda 950 UV/Vis/NIR spectrometer. Emission spectra were recorded on an Edinburgh Instrument FLS920 spectrofluorometer equipped with both continuous $(450 \mathrm{~W})$ xenon and pulsed flash lamps. Thermogravimetric analyses (TGA) was determined by STA449C (NETZSCH). MTT assay was measured on a multimodal microplate reader (Synergy 4, BioTek). The cell imaging was performed with a Leica SP5 laser scanning confocal fluorescence microscope.

\section{Results and discussion}

\section{Characterization of the MSN@C-dots/RB nanoparticles}

The MSN@C-dots/RB nanoparticles were prepared for cell imaging, drug release, and photodynamic therapy, as shown in the schematic illustration (Fig. 1). As shown in Fig. 1, C-dots, MSN@C-dots, MSN@C-dots/RB and the drug loading MSN@C-dots/RB nanoparticles were prepared sequentially. Cdots were prepared using solvent-thermal method, ${ }^{35}$ and the average particle size was proved to be $3.9 \pm 0.8 \mathrm{~nm}$ (Fig. 2a and d). With C-dots embedded in the mesoporous silica (MS) matrix, MSN@C-dots nanoparticles had stable structure and uniform spherical morphology with a diameter of about $79.1 \pm$ $2.7 \mathrm{~nm}$ (Fig. 2b and e), and the worm-like mesoporous channels extending to the surface of the nanoparticles can be clearly observed in the nanoparticles. The energy-dispersive X-ray spectroscopy (EDX) of the MSN@C-dots nanoparticles showed

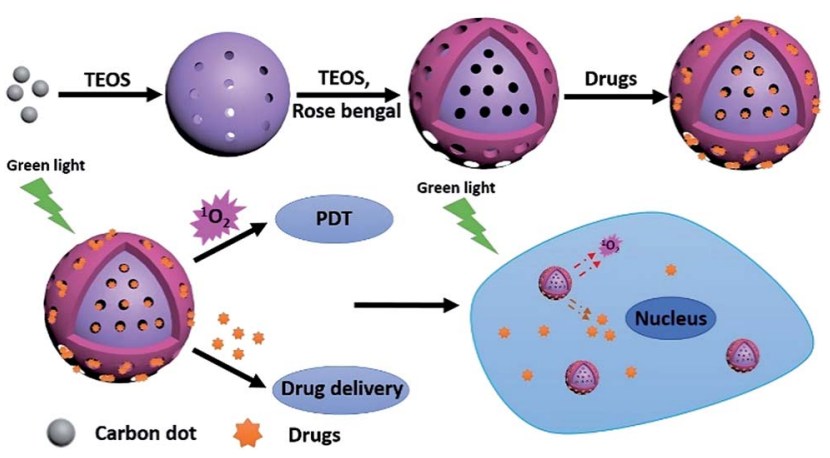

Fig. 1 Illustration of the multifunctional nanoplatform based on MSNs. 


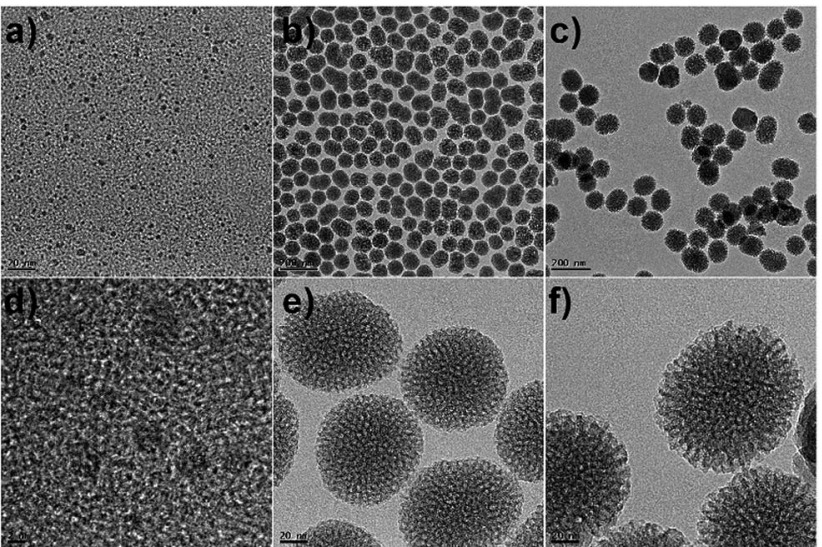

Fig. 2 TEM images of C-dots (a, d), MSNaC-dots nanoparticles (b, e) and MSNaC-dots/RB nanoparticles (c, f).

that the content of carbon element in MSN@C-dots nanoparticles was significantly higher than the MSNs, demonstrating success embedding of C-dots in the MSNs (Fig. S1†). According to thermogravimetric analysis (TGA), the content of C-dots in the MSN@C-dots nanoparticles was quantified to be $0.41 \%(\mathrm{w} / \mathrm{w})$ (Fig. $\mathrm{S} 2 \dagger$ ). It should be noted that the uniform morphologies of MSN@C-dots can not be obtained at the higher doping concentration of C-dots (Fig. S3 $\dagger$ ). In order to achieve the PDT efficacy and more drug-loaded capacity, a mesoporous silica shell was grown on MSN@C-dots nanoparticles for the photosensitizer RB embedding and drug loading. Then, RB was embedded in mesoporous silica shell by epitaxial growth of the MS shell surrounding the MSN@C-dots and the MSN@C-dots/ RB nanoparticles were achieved. As shown in Fig. $2 \mathrm{c}$ and $\mathrm{f}$, the resulting MSN@C-dots/RB nanoparticles have the coreshell structures, and their average size is $104.2 \pm 6.0 \mathrm{~nm}$. In comparison with MSN@C-dots nanoparticles, the hydrodynamic diameter of MSN@C-dots/RB nanoparticles increased from $78.8 \mathrm{~nm}$ to $105.7 \mathrm{~nm}$ (Fig. S4 $\dagger$ ), owing to the mesoporous silica shell was epitaxial grown on MSN@C-dots nanoparticles. Compared with MSN@C-dots nanoparticles, thermogravimetric analysis (TGA) of the MSN@C-dots/RB nanoparticles showed more weight loss and different decomposition temperature, because the RB embedded in MSN@C-dots/RB nanoparticles (Fig. S2 $\dagger$ ). The content of RB in the MSN@C-dots/RB nanoparticles was determined to be $0.54 \%(\mathrm{w} / \mathrm{w})$. These results suggest the mesoporous silica shell successfully epitaxial growing on MSN@C-dots nanoparticles and RB successfully embedding in the mesoporous silica shell.

The mesoporous structure of MSN@C-dots/RB nanoparticles were further characterized by $\mathrm{N}_{2}$ sorption technique. Fig. 3 showed the $\mathrm{N}_{2}$ adsorption-desorption isotherms and the corresponding pore size distributions of MSN@C-dots nanoparticles and MSN@C-dots/RB nanoparticles. The MSNs exhibit type IV isotherm, suggesting that the MSNs have mesoporous structure. ${ }^{18}$ After epitaxial growing of a mesoporous silica shell, the $\mathrm{N}_{2}$ adsorption amount of the nanoparticles achieved a significant increase. The pore volume increased from 0.3329 $\mathrm{cm}^{3} \mathrm{~g}^{-1}$ to $0.4003 \mathrm{~cm}^{3} \mathrm{~g}^{-1}$ and BET surface area increased from
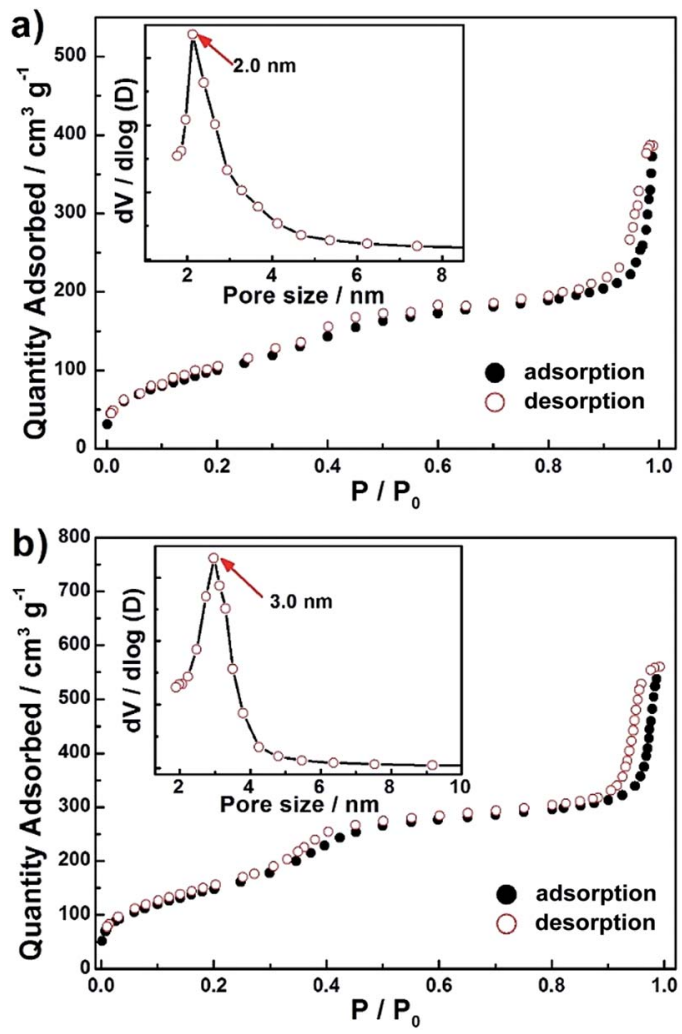

Fig. $3 \quad \mathrm{~N}_{2}$ adsorption-desorption isotherms and pore size distribution curve (inset) of MSNaC-dots nanoparticles (a) and MSNaC-dots/RB nanoparticles (b).

$170.8866 \mathrm{~m}^{2} \mathrm{~g}^{-1}$ to $379.1326 \mathrm{~m}^{2} \mathrm{~g}^{-1}$, respectively. In the meantime, the mesopore average size increased from 2.0 to $3.0 \mathrm{~nm}$. The increase in pore volume, surface area, and mesopore size indicated the potentially enhanced drug loading capacity of MSN@C-dots/RB nanoparticles compared with MSN@C-dots nanoparticles.

\section{Optical properties and loading capacity of the MSN@C-dots/ RB nanoparticles}

The optical properties of the as-prepared nanoparticles had been further explored. As shown in Fig. 4a, MSN@C-dots nanoparticles showed distinct excitation-independent photoluminescence property, which was similar to the pure C-dots and indicated the C-dots were homogeneously embedded in the MSN@C-dots. ${ }^{23}$ The fluorescence emission of C-dots may be surface-state emission, where the bandgap is affected by the particle size and surface properties of C-dots ${ }^{35,55}$ After epitaxial growing a mesoporous silica shell on MSN@C-dots nanoparticles, MSN@C-dots/RB nanoparticles show a broad emission band ranged from $570 \mathrm{~nm}$ to $660 \mathrm{~nm}$, and RB emission peak and intensity was nearly unchanged (Fig. 4b), which indicated the interaction between C-dots and RB was relatively weak.

In order to evaluate the RB loading capability and the stability of MSN@C-dots/RB nanoparticles, we performed a loading and release study of the RB in MSN@C-dots/RB 

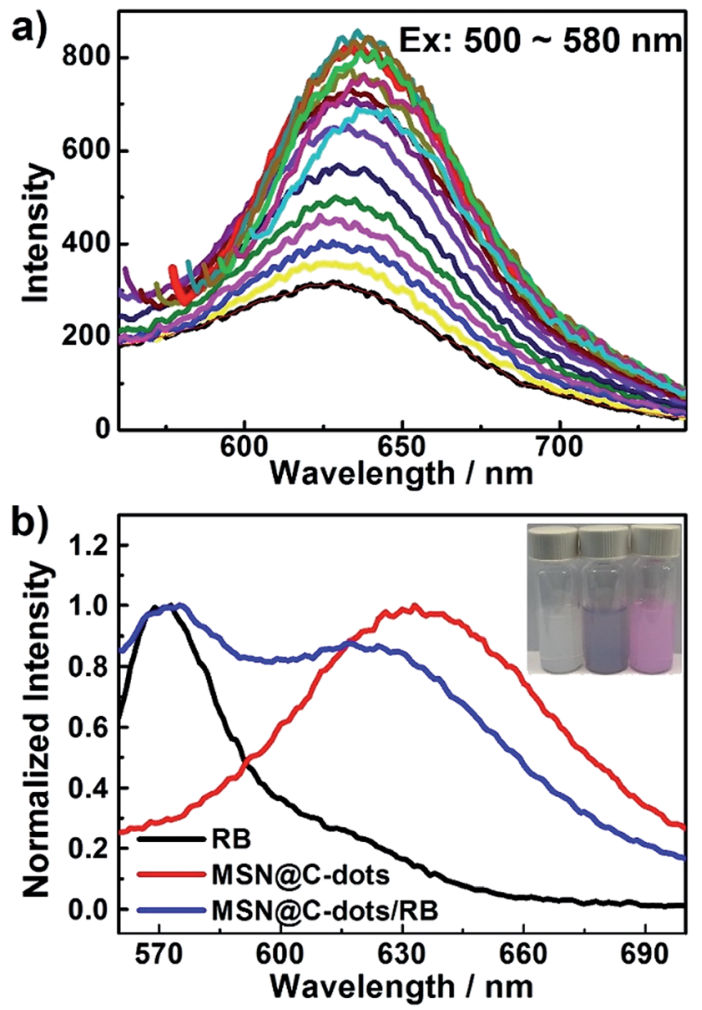

Fig. 4 (a) The emission spectra of MSNaC-dots nanoparticles under different excitation. (b) The emission spectra of RB (black line), MSN@C-dots nanoparticles (red line) and MSNaC-dots/RB nanoparticles (blue line). The insets (from left to right) show photographs of MSNs, MSNaC-dots nanoparticles and MSNaC-dots/RB nanoparticles dispersed in aqueous solution.

nanoparticles. From the PL spectrum of MSN@C-dots/RB nanoparticles soaked in phosphate-buffered saline (PBS, $0.25 \mathrm{mg} \mathrm{mL}^{-1}$ ) (Fig. S5 $\dagger$ ), the content of RB in the MSN@C-dots/ $\mathrm{RB}$ nanoparticles was quantified to be $0.52 \%(\mathrm{w} / \mathrm{w})$, which is consistent with the TGA result. Moreover, it was found that a negligibly low content of RB was released from MSN@C-dots/ $\mathrm{RB}$ nanoparticles even for $72 \mathrm{~h}$ soaked in PBS buffer with varying $\mathrm{pH}(5.0,6.0,6.5,7.0)$ (Fig. S6†), which verifies the high stability of the MSN@C-dots/RB nanoparticles. The cytotoxicity of MSN@C-dots/RB nanoparticles was measured on human embryo lung fibroblast cell line (HELF) by using a MTT assay. The cell viability was determined to be higher than $90 \%$ even at a high concentration of $100 \mu \mathrm{g} \mathrm{mL}^{-1}$ for MSN@C-dots/RB nanoparticles (Fig. S7†). The high cell viability infers that MSN@C-dots/RB is biocompatible and nearly nontoxic to cells.

Then, the drug loading and delivery capability of MSN@Cdots/RB nanoparticles was demonstrated by the $\mathrm{pH}^{-}$ dependent DOX release. From the UV-Vis spectra of the DOX solution (Fig. S8 $\dagger$ ), the content of DOX loading in the MSN@Cdots/RB nanoparticles was quantified to be $34.4 \%(\mathrm{w} / \mathrm{w})$ and the loading efficiency of DOX was 82.6\%. Notably, MSN@C-dots/RB nanoparticles have ultralow drug cumulative release amount at neutral condition, exhibiting a low value of about $17.9 \%$ over the course of $24 \mathrm{~h}$. A substantial increase of drug release rate was observed in acidic solutions, the cumulative release of
MSN@C-dot/RB nanoparticles reached about 50.5\% at pH 5.0 over the course of $24 \mathrm{~h}$ (Fig. S9†). Due to acidic extracellular microenvironment around tumour, the above $\mathrm{pH}$-dependent release properties of MSN@C-dots/RB nanoparticles were favorable for increasing the cancer therapy efficacy and reducing the side effects.

\section{In vitro anticancer effect and cell imaging of the MSN@C- dots/RB nanoparticles}

To explore the PDT effect of MSN@C-dots/RB nanoparticles, the production of ${ }^{1} \mathrm{O}_{2}$ in MSN@C-dots/RB nanoparticles was firstly detected by measuring the bleaching of 1,3-diphenylisobenzofuran (DPBF), whose absorbance at $415 \mathrm{~nm}$ would be diminished in the presence of ${ }^{1} \mathrm{O}_{2}$. As shown in Fig. 5a, the absorbance of DPBF remained nearly unchanged in the control experiments (MSN@C-dots nanoparticles with light irradiation or MSN@C-dots/RB nanoparticles without light irradiation), indicating the absence of ${ }^{1} \mathrm{O}_{2}$ production. By contrast, the absorbance of DPBF incubated with MSN@C-dots/RB nanoparticles decreased exponentially with the time under $540 \mathrm{~nm}$ light irradiation at a power of $300 \mathrm{~mW} \mathrm{~cm}^{-2}$ and decreased approximately $70 \%$ in $10 \mathrm{~min}$, indicating a high ${ }^{1} \mathrm{O}_{2}$ production.
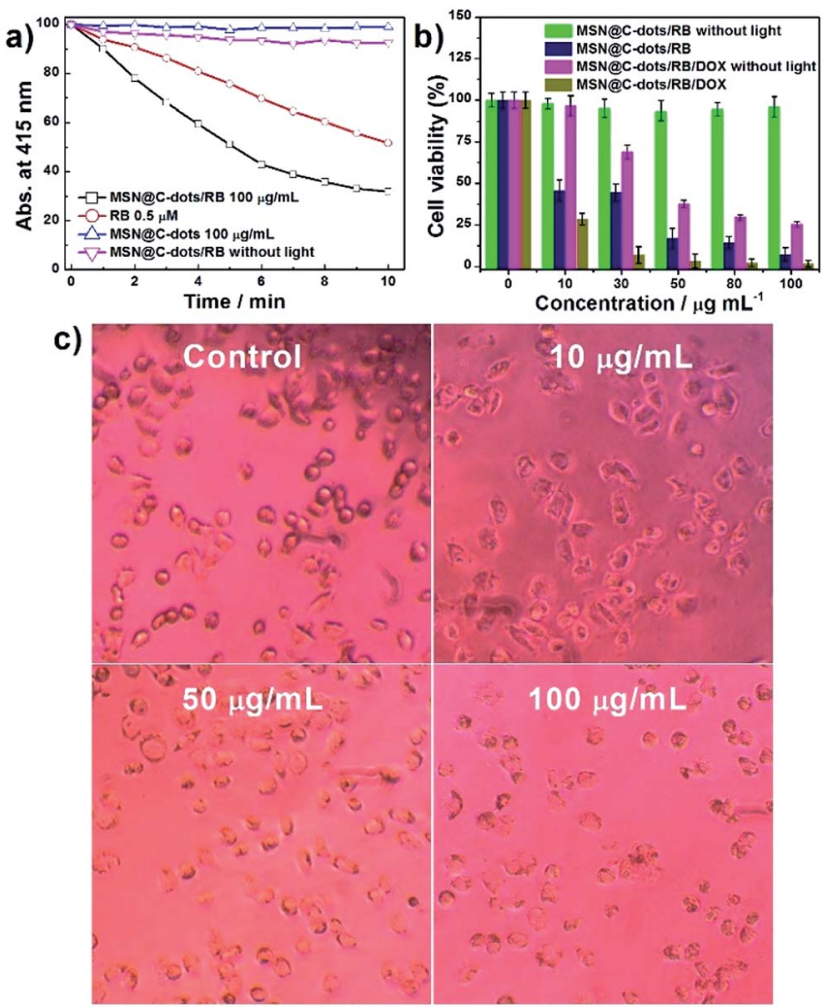

Fig. 5 (a) Time-dependent bleaching of DPBF caused by ${ }^{1} \mathrm{O}_{2}$ generation in the presence of MSNaC-dots/RB nanoparticles under $540 \mathrm{~nm}$ light irradiation. All the absorbances were normalized at the zero point of irradiation time. (b) The cell viability of $\mathrm{H} 1299$ cells treated with different concentrations of MSNaC-dots/RB nanoparticles and MSNaC-dots/RB/DOX nanoparticles with or without green light irradiation. (c) The corresponding microscopic images of H1299 cells treated with different concentrations of MSNaC-dots/RB nanoparticles under green light irradiation. 
These results unambiguously demonstrated that the generation of ${ }^{1} \mathrm{O}_{2}$ was triggered by MSN@C-dots/RB nanoparticles upon green light excitation. Notably, compared with the same amount of RB solution, ${ }^{1} \mathrm{O}_{2}$ production of MSN@C-dots/RB nanoparticles was about 1.4 times higher under light irradiation (Fig. 5a). The excellent ${ }^{1} \mathrm{O}_{2}$ production efficiency of the MSN@C-dots/RB nanoparticles is due to the mesoporous silica matrix can effectively inhibit the self-aggregation of RB and the core/shell structure decrease the interaction of excited RB and C-dots.

In this study, in vitro cytotoxicity of the MSN@C-dots/RB nanoparticles to cancer cells H1299 using a MTT assay was investigated to evaluate PDT efficiency of this MSNs based nanoplatform. As shown in Fig. 5b, upon $540 \mathrm{~nm}$ light irradiation at a relatively low power of $300 \mathrm{~mW} \mathrm{~cm} \mathrm{cor}^{-2}$ for $\mathrm{min}$, a significant reduction in cell viability was observed for H1299 cells incubated with MSN@C-dots/RB nanoparticles, in stark contrast to those incubated with MSN@C-dots/RB nanoparticles without light irradiation. The corresponding microscopic images of H1299 cells clearly showed the remarkable changes of cell morphology and an increased cell death with the increasing concentration of MSN@C-dots/RB nanoparticles (Fig. 5c), which were consistent with the results of cell viability acquired from MTT assays.

To investigate the synergistic effects of PDT with chemotherapy, three control groups (MSN@C-dots/DOX, MSN@Cdots/RB/DOX without light irradiation, MSN@C-dots/RB with light irradiation) were carried out to treat with H1299 cells, followed by the MTT assay test. The mono-therapy groups, MSN@C-dots/DOX and MSN@C-dots/RB/DOX without light irradiation, exhibited moderate cytotoxicity to H1299 cells (Fig. S10 $†$ and 5b). In addition, the trend of the reduction that H1299 cells incubated with MSN@C-dots/RB/DOX nanoparticles without light irradiation was relatively faster than MSN@C-dots/DOX group, illustrating the high DOX loading capacity of MSN@C-dots/RB nanocomposites (Fig. S10†). Furthermore, by employing the combined therapy group (MSN@C-dot/RB/DOX with light irradiation), the greatest reduction in cell viability was observed. Specifically, only $6.8 \%$ cell viability of $\mathrm{H} 1299$ cells was tested at the concentration of 30 $\mu \mathrm{g} \mathrm{mL}^{-1}$ MSN@C-dot/RB/DOX nanoparticles (Fig. 5b). The combined therapy group exhibited a remarkably improved therapy relative to that of single chemotherapy or PDT group. These results confirm the high therapeutic efficacy of MSN@C$\mathrm{dot} / \mathrm{RB} / \mathrm{DOX}$ nanoparticles in vitro, and thus show great promise of developing MSN@C-dot/RB/DOX nanoparticles as an efficient anticancer agent in biological or clinical applications.

Besides chemo/photodynamic therapy, the MSN@C-dots/RB nanoparticles could be also used for cancer cell imaging by utilizing their intense photoluminescence. After incubation with H1299 cells, the red emission of C-dots from the MSN@Cdots/RB nanoparticles was clearly visualized in the cytoplasm upon $540 \mathrm{~nm}$ light irradiation (Fig. 6 a). These results show that the MSNs-based nanoplatform may serve as a fluorescence imaging agent for imaging-guided synergistic chemotherapy and PDT therapy, where the photoluminescence of the C-dots can be used to monitor cancer cells, the photosensitizer can

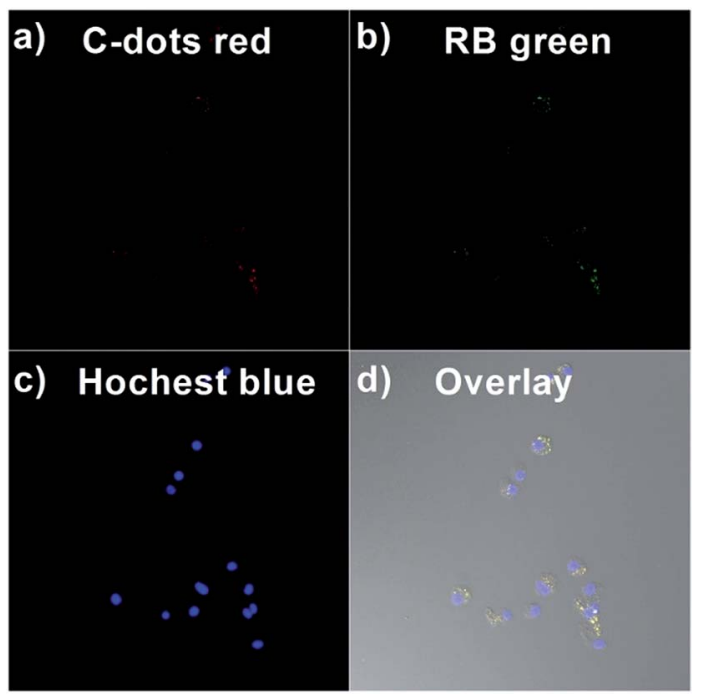

Fig. 6 Confocal microscopy images of $\mathrm{H} 1299$ cells incubated with MSNaC-dots/RB nanoparticles for $2 \mathrm{~h}$ at $37^{\circ} \mathrm{C}$. (a) The $\mathrm{C}$-dots red emission image of MSNaC-dots/RB nanoparticles $\left(\lambda_{\mathrm{em}}=590-\right.$ $680 \mathrm{~nm}, \lambda_{\mathrm{ex}}=552 \mathrm{~nm}$ ), (b) the RB green emission image of MSN@Cdots/RB nanoparticles $\left(\lambda_{\mathrm{em}}=565-590 \mathrm{~nm}, \lambda_{\mathrm{ex}}=552 \mathrm{~nm}\right)$ and (c) the Hochest blue emission image MSNaC-dots/RB nanoparticles $\left(\lambda_{\mathrm{em}}=\right.$ 450-490 nm, $\lambda_{\text {ex }}=405 \mathrm{~nm}$ ). (d) The overlay image of (a)-(c).

be used to generate ${ }^{1} \mathrm{O}_{2}$ for photodynamic therapy, and the mesoporous structure can be utilized to loading drugs for chemotherapy.

\section{Antimicrobial activity of the MSN@C-dots/RB nanoparticles}

Since frequently the bacterial infection contamination accompanying cancer progression can injure the immune system and deteriorate cancers, bacterial inhibition ability of MSN@C-dots/ $\mathrm{RB}$ nanoparticles also had been investigated. To explore the bacterial inhibitory effect, we researched $E$. coli growth kinetics in liquid Luria-Bertani (LB) media by measuring the optical density (OD) at $600 \mathrm{~nm}$ of the bacterial suspensions. Fig. 7a illustrated the values of the $\mathrm{OD}_{600}$ that $E$. coli treated with different concentrations of MSN@C-dots/RB nanoparticles with green light irradiation at different incubation times. With increasing the concentrations of MSN@C-dots/RB nanoparticles, the growth of $E$. coli was progressively inhibited, which indicated MSN@C-dots/RB nanoparticles have a dosedependent manner at bacterial inhibiting. When the concentration of MSN@C-dots/RB nanoparticles reached to $100 \mu \mathrm{g}$ $\mathrm{mL}^{-1}$, the bacterial growth of $E$. coli was delayed about $12 \mathrm{~h}$ under green light irradiation. To further explore MSN@C-dots/ $\mathrm{RB}$ nanoparticles as a nanocarrier's platform, the effect of MSN@C-dots/RB nanoparticles was investigated by loading an antibacterial agent of ampicillin (Amp) in MSN@C-dots/RB nanoparticles (loading capacity: 18.3\% (w/w), loading efficiency: $29.3 \%$, Fig. S11 $\dagger$ ). The antibacterial effect of such platform (MSN@C-dots/RB/Amp) without light irradiation were firstly investigated. As shown in Fig. S12, $\uparrow$ the growth of $E$. coli was gradually suppressed with increasing MSN@C-dots/RB/ Amp concentration, illustrating the antibacterial effect of 

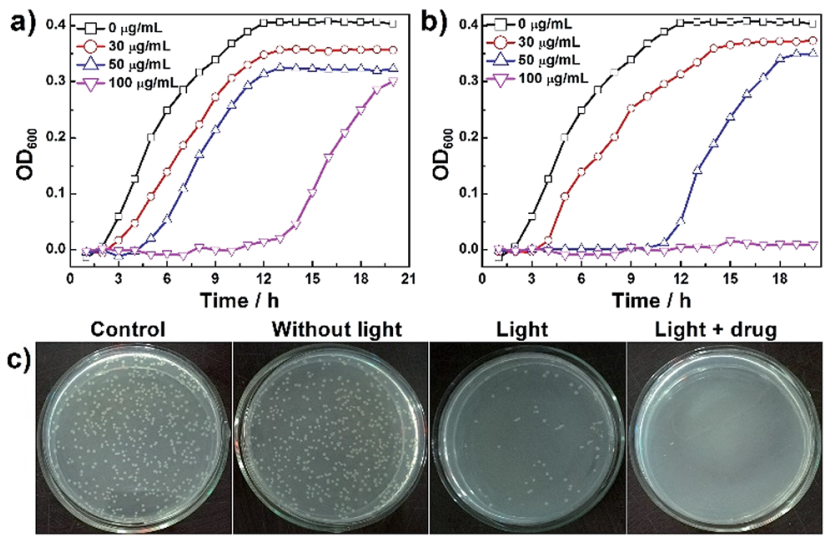

Fig. 7 (a) The growth curve of E. coli treated with different concentrations of MSNaC-dots/RB under green light irradiation. (b) The growth curve of $E$. coli treated with different concentrations of MSNaC-dots/RB-Amp under green light irradiation. (c) The photographs of $E$. coli colonies formed on LB-agar plates after treatments with MSNaC-dots/RB nanoparticles. From left to right: control, MSNaC-dots/RB without light irradiation, MSNaC-dots/RB with light irradiation and MSNaC-dots/RB/Amp with light irradiation.

Amp. In comparison, MSN@C-dots/RB/Amp with light irradiation group exhibited more remarkable inhibitory effect, and the growth of $E$. coli was significantly suppressed with otherwise identical conditions (Fig. 7b). When the concentration of MSN@C-dots/RB/Amp nanoparticles reached to $100 \mu \mathrm{g} \mathrm{mL}{ }^{-1}$, the $E$. coli were almost completely killed. These results reveal great potential of the MSN@C-dot/RB/Amp nanoparticles as an dual-mole antibacterial agents for antimicrobial treatment.

The agar plate experiments of $E$. coli were also evaluated the antibacterial effect of MSN@C-dots/RB nanoparticles. The concentration of MSN@C-dots/RB nanoparticles was $100 \mu \mathrm{g}$ $\mathrm{mL}^{-1}$. The photos of bacterial colonies clearly displayed on the corresponding agar plates and indicated the differences in bacterial inhibiting effects (Fig. 7c). Compared with the control plate, the E. coli incubated with MSN@C-dots/RB nanoparticles without light irradiation showed little difference in the number of $E$. coli colonies indicating MSN@C-dots/RB nanoparticles cannot inhibit bacteria by themselves. In contrast, the number of $E$. coli colonies showed significant reduction under green light irradiation, which confirmed the antibacterial effect of MSN@C-dots/RB nanoparticles was obtained by the generation of ${ }^{1} \mathrm{O}_{2}$ under light irradiation (Fig. 7c). For the E. coli treated with MSN@C-dots/RB/Amp nanoparticles under the green light irradiation, there was no visible bacterial colonies lawn on the plate suggesting the complete inhibiting effect at $100 \mu \mathrm{g} \mathrm{mL}{ }^{-1}$. These results demonstrated MSN@C-dots/RB nanoparticles could effectively inhibit the growth of $E$. coli under light irradiation and hold great potential in the bacteria inhibiting field.

\section{Conclusions}

In summary, we have developed a general strategy to fabricate a multifunctional nanoplatform MSN@C-dots/RB, in which carbon dots embedded in MSNs core, photosensitizers RB doped in mesoporous silica shell and the drugs loaded in mesoporous silica channel. Core/shell structure of MSNs has been smartly designed to prevent the self-aggregation of carbon dots and photosensitizers, avoid the mutual interference between them, and increase the loading capacity of drugs. All of these advantages are critical for improving cancer therapy efficacy than that of single chemotherapy or PDT group. In particular, compared with the pure RB, 1.4 times higher ${ }^{1} \mathrm{O}_{2}$ production has been achieved in our multifunctional nanoplatform upon $10 \mathrm{~min}$ light irradiation. And the enhanced anticancer efficacy has been obtained by the combined chemo/ photodynamic therapy. More than $90 \%$ reduction in cell viability has been achieved at the concentration of $30 \mu \mathrm{g} \mathrm{mL}$ MSN@C-dot/RB/DOX nanoparticles under light irradiation. Moreover, by taking advantages of the red PL of the carbon dots embedded in MSN@C-dots/RB, we have demonstrated their potential as a nanoplatform for imaging-guided chemo/ photodynamic therapy. Finally, we have shown the high efficacy of the MSN@C-dots/RB for the inhibition of bacteria. Therefore, the MSN@C-dots/RB nanoparticles are a promising multifunctional nanoplatform for enhancing the therapeutic efficiency in medicine therapy.

\section{Acknowledgements}

We greatly thank the financial supports from the National Nature Science Foundation of China (31270790, 31300650 and 31470741), the National Thousand Talents Program of China, and China Postdoctoral Science Foundation (2016M592096). We wish to thank Dr Lei Zhang for many helpful discussions.

\section{References}

1 L. Cheng, C. Wang, L. Feng, K. Yang and Z. Liu, Chem. Rev., 2014, 114, 10869-10939.

2 T. Lammers, F. Kiessling, W. E. Hennink and G. Storm, Mol. Pharm., 2010, 7, 1899-1912.

3 J. Kim, Y. Piao and T. Hyeon, Chem. Soc. Rev., 2009, 38, 372390.

4 J. P. Celli, B. Q. Spring, I. Rizvi, C. L. Evans, K. S. Samkoe, S. Verma, B. W. Pogue and T. Hasan, Chem. Rev., 2010, 110, 2795-2838.

5 H. Gong, Z. Dong, Y. Liu, S. Yin, L. Cheng, W. Xi, J. Xiang, K. Liu, Y. Li and Z. Liu, Adv. Funct. Mater., 2014, 24, 64926502.

6 Z. Zhang, L. Wang, J. Wang, X. Jiang, X. Li, Z. Hu, Y. Ji, X. Wu and C. Chen, Adv. Mater., 2012, 24, 1418-1423.

7 S. S. Lucky, N. Muhammad Idris, Z. Li, K. Huang, K. C. Soo and Y. Zhang, ACS Nano, 2015, 9, 191-205.

8 K. Lu, C. He, N. Guo, C. Chan, K. Ni, R. R. Weichselbaum and W. Lin, J. Am. Chem. Soc., 2016, 138, 12502-12510.

9 Y. Wang, H. Wang, D. Liu, S. Song, X. Wang and H. Zhang, Biomaterials, 2013, 34, 7715-7724.

10 B. Sun, B. Ranganathan and S.-S. Feng, Biomaterials, 2008, 29, 475-486.

11 F. Tang, L. Li and D. Chen, Adv. Mater., 2012, 24, 1504-1534. 
12 J.-N. Liu, W.-B. Bu and J.-L. Shi, Acc. Chem. Res., 2015, 48, 1797-1805.

13 N. Erathodiyil and J. Y. Ying, Acc. Chem. Res., 2011, 44, 925935.

14 H. Meng, M. Xue, T. Xia, Z. Ji, D. Y. Tarn, J. I. Zink and A. E. Nel, ACS Nano, 2011, 5, 4131-4144.

15 P. Yang, S. Gai and J. Lin, Chem. Soc. Rev., 2012, 41, 36793698.

16 E.-K. Lim, T. Kim, S. Paik, S. Haam, Y.-M. Huh and K. Lee, Chem. Rev., 2015, 115, 327-394.

17 L. Zhang, Y. Chen, Z. Li, L. Li, P. Saint-Cricq, C. Li, J. Lin, C. Wang, Z. Su and J. I. Zink, Angew. Chem., Int. Ed., 2016, 128, 2158-2161.

18 Y.-S. Lin, S.-H. Wu, Y. Hung, Y.-H. Chou, C. Chang, M.-L. Lin, C.-P. Tsai and C.-Y. Mou, Chem. Mater., 2006, 18, 5170-5172.

19 L. Li, F. Tang, H. Liu, T. Liu, N. Hao, D. Chen, X. Teng and J. He, ACS Nano, 2010, 4, 6874-6882.

20 I. Slowing, B. G. Trewyn and V. S.-Y. Lin, J. Am. Chem. Soc., 2006, 128, 14792-14793.

21 E. Gandin, Y. Lion and A. Van de Vorst, Photochem. Photobiol., 1983, 37, 271-278.

22 E. Gianotti, B. Martins Estevão, F. Cucinotta, N. Hioka, M. Rizzi, F. Renò and L. Marchese, Chem.-Eur. J., 2014, 20, 10921-10925.

23 J. Gehring, B. Trepka, N. Klinkenberg, H. Bronner, D. Schleheck and S. Polarz, J. Am. Chem. Soc., 2016, 138, 3076-3084.

24 H. Liu, Y. Yang, A. Wang, M. Han, W. Cui and J. Li, Adv. Funct. Mater., 2016, 26, 2561-2570.

25 A. Yildirim, M. Turkaydin, B. Garipcan and M. Bayindir, RSC Adv., 2016, 6, 32060-32069.

26 J. Zhan, Z. Ma, D. Wang, X. Li, X. Li, L. Le, A. Kang, P. Hu, L. She and F. Yang, Int. J. Nanomed., 2017, 12, 2733.

27 J. G. Croissant, X. Cattoën, J.-O. Durand, M. W. C. Man and N. M. Khashab, Nanoscale, 2016, 8, 19945-19972.

28 G. Yang, H. Gong, X. Qian, P. Tan, Z. Li, T. Liu, J. Liu, Y. Li and Z. Liu, Nano Res., 2015, 8, 751-764.

29 M. Gary-Bobo, Y. Mir, C. Rouxel, D. Brevet, I. Basile, M. Maynadier, O. Vaillant, O. Mongin, M. BlanchardDesce, A. Morère, M. Garcia, J. O. Durand and L. Raehm, Angew. Chem., Int. Ed., 2011, 123, 11627-11631.

30 D. Brevet, M. Gary-Bobo, L. Raehm, S. Richeter, O. Hocine, K. Amro, B. Loock, P. Couleaud, C. Frochot and A. Morère, Chem. Commun., 2009, 1475-1477.

31 T. Wang, L. Zhang, Z. Su, C. Wang, Y. Liao and Q. Fu, ACS Appl. Mater. Interfaces, 2011, 3, 2479-2486.

32 D. Shen, J. Yang, X. Li, L. Zhou, R. Zhang, W. Li, L. Chen, R. Wang, F. Zhang and D. Zhao, Nano Lett., 2014, 14, 923932.
33 S. Y. Lim, W. Shen and Z. Gao, Chem. Soc. Rev., 2015, 44, 362381.

34 P. Miao, K. Han, Y. Tang, B. Wang, T. Lin and W. Cheng, Nanoscale, 2015, 7, 1586-1595.

35 S. Qu, D. Zhou, D. Li, W. Ji, P. Jing, D. Han, L. Liu, H. Zeng and D. Shen, Adv. Mater., 2016, 28, 3516-3521.

36 L. Cao, X. Wang, M. J. Meziani, F. Lu, H. Wang, P. G. Luo, Y. Lin, B. A. Harruff, L. M. Veca, D. Murray, S.-Y. Xie and Y.-P. Sun, J. Am. Chem. Soc., 2007, 129, 11318-11319.

37 S. N. Baker and G. A. Baker, Angew. Chem., Int. Ed., 2010, 49, 6726-6744.

38 Y. Liu, Y. Tian, Y. Tian, Y. Wang and W. Yang, Adv. Mater., 2015, 27, 7156-7160.

39 X. Jia, M. Pei, X. Zhao, K. Tian, T. Zhou and P. Liu, ACS Biomater. Sci. Eng., 2016, 2, 1641-1648.

40 Q. Wang, S. Zhang, Y. Zhong, X.-F. Yang, Z. Li and H. Li, Anal. Chem., 2017, 89, 1734-1741.

41 Y. Wang and A. Hu, J. Mater. Chem. C, 2014, 2, 6921-6939.

42 Y. Chen, M. Zheng, Y. Xiao, H. Dong, H. Zhang, J. Zhuang, H. Hu, B. Lei and Y. Liu, Adv. Mater., 2016, 28, 312-318.

43 D. Zhou, D. Li, P. Jing, Y. Zhai, D. Shen, S. Qu and A. L. Rogach, Chem. Mater., 2017, 29, 1779-1787.

44 T. H. Kim, F. Wang, P. McCormick, L. Wang, C. Brown and Q. Li, J. Lumin., 2014, 154, 1-7.

45 Z. Xie, F. Wang and C. y. Liu, Adv. Mater., 2012, 24, 17161721.

46 F. Wang, Z. Xie, H. Zhang, C. y. Liu and Y. g. Zhang, Adv. Funct. Mater., 2011, 21, 1027-1031.

47 Y. Chen, B. Lei, M. Zheng, H. Zhang, J. Zhuang and Y. Liu, Nanoscale, 2015, 7, 20142-20148.

48 Y. Liu, C.-y. Liu, Z.-y. Zhang, W.-d. Yang and S.-d. Nie, J. Mater. Chem. C, 2015, 3, 2881-2885.

49 X. Liu, N. Zhang, T. Bing and D. Shangguan, Anal. Chem., 2014, 86, 2289-2296.

50 C. Liu, L. Bao, B. Tang, J. Y. Zhao, Z. L. Zhang, L. H. Xiong, J. Hu, L. L. Wu and D. W. Pang, Small, 2016, 12, 4702-4706.

51 C. Cheng, X. Tan, D. Lu, L. Wang, T. Sen, J. Lei, A. M. El-Toni, J. Zhang, F. Zhang and D. Zhao, Chem.-Eur. J., 2015, 21, 17944-17950.

52 R. Liu, D. Wu, S. Liu, K. Koynov, W. Knoll and Q. Li, Angew. Chem., Int. Ed., 2009, 121, 4668-4671.

53 L. Zhou, Z. Li, Z. Liu, J. Ren and X. Qu, Langmuir, 2013, 29, 6396-6403.

54 G. Bucaneve, A. Micozzi, F. Menichetti, P. Martino, M. S. Dionisi, G. Martinelli, B. Allione, D. D'antonio, M. Buelli and A. M. Nosari, N. Engl. J. Med., 2005, 353, 977-987.

55 L. Bao, C. Liu, Z.-L. Zhang and D.-W. Pang, Adv. Mater., 2015, 27, 1663-1667. 